\title{
Spatial components dependence for bidimensional time-constant AR(1) model with $\alpha$-stable noise and triangular coefficients matrix
}

\author{
Aleksandra Grzesiek ${ }^{1}$ (D)
}

Accepted: 29 July 2021 / Published online: 3 September 2021

(C) The Author(s) 2021

\begin{abstract}
In this paper, we examine the bidimensional time-constant autoregressive model of order 1 with $\alpha$ stable noise. We focus on the case of the triangular coefficients matrix for which one of the spatial components of the model simplifies to the one-dimensional autoregressive time series. We study the asymptotic behaviour of the cross-codifference and the cross-covariation applied to describe the dependence in time between the spatial components of the model. As a result, we formulate the theorem about the asymptotic relation between both measures, which is consistent with the result that is correct for the case of the non-triangular coefficients matrix.
\end{abstract}

Keywords $\alpha$-Stable distribution - Autoregressive model . Codifference $\cdot$ Covariation $\cdot$ Asymptotic behaviour

Mathematics Subject Classification $62 \mathrm{M} 10 \cdot 60 \mathrm{G} 20$

\section{Introduction}

The univariate autoregressive (AR) processes, introduced in the 20s of the previous century by Yule and Slutsky, are the most commonly used models in time series analysis. One of their first applications was presented by Yule in 1927 to model the sunspot time series [1]. Autoregressive time series belong to the class of the short memory processes characterized by exponentially decreasing auto-

Aleksandra Grzesiek

aleksandra.grzesiek@pwr.edu.pl

1 Faculty of Pure and Applied Mathematics, Hugo Steinhaus Center, Wrocław University of Science and Technology, Wybrzeże Wyspiańskiego 27, 50-370 Wrocław, Poland correlation. The univariate AR models, describing a single variable, are commonly generalized to the multivariate autoregressive time series. They can model the multivariate data with not only interdependence within each spatial component, but also the mutual dependence between different spatial components [2-4].

In this paper, we consider the bidimensional autoregressive model which is based on the non-Gaussian symmetric $\alpha$-stable noise. Such an extension of the classical Gaussian model can be found in many scientific articles regarding univariate and multivariate AR processes [5-15]. We remind here that the $\alpha$-stable distribution with $0<\alpha<2$ is an example of the heavy-tailed distributions for which the tails are not exponentially bounded. Due to the generalized central limit theorem, the $\alpha$-stable distribution is the only non-degenerated limiting distribution of a sum of independent identically distributed random variables with infinite variance. We mention here that the $\alpha$-stable distribution was introduced by Lévy and Khinchine in $[16,17]$ and the standardized mathematical background, which contributed to the increased interest in the $\alpha$-stable-based models, was provided by Gnedenko and Kolomogorov [18], Feller [19], Zolotarev [20], Weron [21], and later in the 1990s Shao and Nikias [22], Janicki and Weron [23] and Samorodnitsky and Taqqu [24]. Since the first significant application provided by Mandelbrot in [25], the $\alpha$ stable distribution is commonly applied in finance and economics [26-33], physics [34-36], signal processing [37-39], computer science [40, 41], geology and geophysics [42, 43], biology [44, 45], and many other fields.

Since for $0<\alpha<2$ the second moment of an $\alpha$ stable random variable is infinite, one cannot use the second-moment-based measures to describe the dependence between $\alpha$-stable random variables. Therefore, in the last decades, the researchers have developed some alternatives 
that can replace the covariance and the correlation in this context. Here, we focus our attention to the covariation and the codifference which are the most frequently used in the literature devoted to the $\alpha$-stable models, see [24, 46-50]. However, there are also other measures that can be applied in this context, e.g. association parameter [51], generalized association parameter [52], symmetric covariation coefficient [53, 54], signed symmetric covariation coefficient $[55,56]$, fractional lower order covariance [57], the Lévy correlation cascade $[58,59]$, the coefficient $R_{G}$ [60], and the distance-based measure proposed in [61].

As a consequence of the above-mentioned fact, for the one-dimensional stochastic processes with $\alpha$-stable finitedimensional distributions, the interdependence in time cannot be described using the auto-covariance or the autocorrelation. Therefore, in the literature, those measures are commonly replaced by the auto-codifference and the autocovariation. In particular, many researchers are interested in examining the way the mentioned measured decay as the lag increases, see for example [24, 62-67]. In the context of the autoregressive models, such considerations are presented by Nowicka for the auto-dependence measures of the one-dimensional $\alpha$-stable ARMA models [7] and by Nowicka and Wyłomańska for the auto-dependence measures for one-dimensional $\alpha$-stable time-periodic AR models of order 1 and one-dimensional $\alpha$-stable timevarying AR models of order $1[68,69]$.

In this paper, we use the mentioned measures to describe the dependence between spatial components of the bidimensional autoregressive model of order 1 with $\alpha$ stable noise. The concept of the so-called cross-dependence was considered in the author's previous papers where the cross-codifference and the cross-covariation of the bidimensional AR(1) model were examined in the case of the time-constant and time-periodic, see [70-74]. Here, we also analyse the time-constant model, but we focus on the case of the triangular coefficients matrix, which was not covered in the mentioned papers.

The article is organized as follows. In Sect. 2, we present the considered model together with the corresponding cross-dependence measures. In Sect. 3, we examine the asymptotics of the cross-codifference and cross-covariation. Section 4 presents the sample illustration of the obtained results. In Sect. 5, we present the summary of the paper.

\section{Bidimensional time-constant AR(1) model with triangular matrix}

In this section, we present the bidimensional time-constant $\mathrm{AR}(1)$ model with $\alpha$-stable noise and a triangular coefficients matrix together with the form of the bounded solution and the corresponding measures of cross-dependence.

\subsection{General bidimensional AR(1) model}

Let us remind that the time series $\left\{\mathbf{X}(t)=\left(X_{1}(t), X_{2}(t)\right)\right\}$ is called the bidimensional autoregressive model of order 1 , if for every $t \in \mathbb{Z}$ the following equation is satisfied [4]

$\mathbf{X}(t)-\boldsymbol{\Theta} \mathbf{X}(t-1)=\mathbf{Z}(t)$,

where $\Theta$ is the real-valued coefficients matrix of size $2 \times 2$ and $\left\{\mathbf{Z}(t)=\left(Z_{1}(t), Z_{2}(t)\right)\right\}$ is a series of uncorrelated (in particular, independent) random vectors. The model satisfying Eq. (1) has the so-called stationary causal representation of the form [4]

$\mathbf{X}(t)=\sum_{j=0}^{+\infty} \boldsymbol{\Theta}^{j} \mathbf{Z}(t-j)$,

assuming that the absolute values of the eigenvalues of $\Theta$ are less than 1 . If this condition is met, $\mathbf{X}(t)$ given in Eq. (2) is bounded for each $t$.

Let us denote the elements of the coefficients matrix as follows

$\boldsymbol{\Theta}=\left[\begin{array}{ll}\Theta_{11} & \Theta_{12} \\ \Theta_{21} & \Theta_{22}\end{array}\right]$.

Moreover, let us assume that all elements of $\boldsymbol{\Theta}$ except one are nonzero and the only zero-element is located outside of the main diagonal. More precisely, we consider the case of the lower-triangular matrix with $\Theta_{12}=0$ of the following form

$\boldsymbol{\Theta}^{(1)}=\left[\begin{array}{cc}\Theta_{11} & 0 \\ \Theta_{21} & \Theta_{22}\end{array}\right]$.

or the upper-triangular matrix with $\Theta_{21}=0$ given as follows

$\boldsymbol{\Theta}^{(2)}=\left[\begin{array}{cc}\Theta_{11} & \Theta_{12} \\ 0 & \Theta_{22}\end{array}\right]$.

In both above-mentioned cases, we can notice that one of the spatial components of the two-dimensional model simplifies to the univariate AR(1) time series. For example, if we assume that the coefficients matrix has the form given in Eq. (4), the value of $X_{1}(t)$ is Eq. (1) depends linearly only on the value of $X_{1}(t-1)$, whereas $X_{2}(t-1)$ is neglected. However, both spatial components $\left\{X_{1}(t)\right\}$ and $\left\{X_{2}(t)\right\}$ can be mutually dependent if the noise components 
$\left\{Z_{1}(t)\right\}$ and $\left\{Z_{2}(t)\right\}$ are dependent. In the following part of the paper, we take the above assumption. Moreover, it is easy to notice that in the case of the triangular coefficients matrix the condition guaranteeing the bounded solution simplifies to the fact that the main diagonal elements of the matrix $\Theta$ are less than 1 in absolute value.

For $n, m=1,2$, let $\Theta_{n m}^{(j)}$ denote the $(n, m)$ element of the matrix $\Theta$ in Eq. (2). Assuming that the coefficients matrix is triangular, i.e. one of the parameters $\Theta_{12}$ or $\Theta_{21}$ is equal to 0 , it is easy to show that

$\Theta_{11}^{(j)}=\Theta_{11}^{j}$,

$\Theta_{12}^{(j)}=$

$\left\{\begin{array}{cc}0 & \text { if } \Theta_{12}=0, \\ {[5 p t] \frac{\Theta_{22}^{j}-\Theta_{11}^{j}}{\Theta_{22}-\Theta_{11}}} & \text { if } \Theta_{12} \neq 0 \text { and } \Theta_{11} \neq \Theta_{22}, \\ {[8 p t] j \Theta^{j-1} \Theta_{12}} & \text { if } \Theta_{12} \neq 0 \text { and } \Theta_{11}=\Theta_{22}=\Theta,\end{array}\right.$

$\Theta_{21}^{(j)}=$

$\left\{\begin{array}{cc}0 & \text { if } \Theta_{21}=0, \\ {[5 p t] \frac{\Theta_{22}^{j}-\Theta_{11}^{j}}{\Theta_{22}-\Theta_{11}}} & \text { if } \Theta_{21} \neq 0 \text { and } \Theta_{11} \neq \Theta_{22}, \\ {[8 p t] j \Theta^{j-1} \Theta_{21}} & \text { if } \Theta_{21} \neq 0 \text { and } \Theta_{11}=\Theta_{22}=\Theta,\end{array}\right.$

$\Theta_{22}^{(j)}=\Theta_{22}^{j}$.

The above-given representation of the elements of $\boldsymbol{\Theta}^{j}$ is used to present the formulas of the cross-dependence measures in the following sections.

\subsection{Two-dimensional $\alpha$-stable distribution}

In the classical version of the AR time series, the noise $\{\mathbf{Z}(t)\}$ is assumed to be the second-order process in $\mathbb{R}^{2}$ or, in particular, to be Gaussian. Here, we extend this model by assuming that $\mathbf{Z}(t)$ for each $t \in \mathbb{Z}$ is a symmetric $\alpha$ stable random vector in $\mathbb{R}^{2}$ with $1<\alpha<2$.

We remind here that due to the Generalized Central Limit Theorem the $\alpha$-stable random variables with $0<\alpha<2$ are commonly considered as the extension of the Gaussian ones which correspond to the case of $\alpha=2$. However, for the non-Gaussian $\alpha$-stable distribution the expected value and the second moment are infinite for all $\alpha<1$ and all $\alpha<2$, respectively. The two-dimensional symmetric $\alpha$-stable random vector, similarly to the univariate one, can be defined using the characteristic function $[24,52,75,76]$. Namely, the random vector $\mathbf{Z}$ in $\mathbb{R}^{2}$ is said to have the symmetric $\alpha$-stable distribution if and only if there exists a unique symmetric finite spectral measure $\boldsymbol{\Gamma}(\cdot)$ on the unit sphere denoted as $S_{2}$ such that [24]

$\mathbb{E}[\exp \{i\langle z, \mathbf{Z}\rangle\}]=\exp \left\{-\int_{S_{2}}|\langle z, \mathbf{s}\rangle|^{\alpha} \boldsymbol{\Gamma}(d s)\right\}$,

where $\langle\cdot, \cdot\rangle$ is the inner product. The spectral measure $\boldsymbol{\Gamma}$ includes the information about scale and skewness of $\mathbf{Z}$, whereas $0<\alpha<2$ is called the stability parameter that regulates the rate at which the distribution tail decays. Both $\alpha$ and $\boldsymbol{\Gamma}$ fully describe the two-dimensional symmetric $\alpha$ stable distribution.

As it was mentioned before, for the non-Gaussian $\alpha$ stable distribution the second moment is infinite, therefore to measure the dependence between the components of such a vector one cannot use the covariance or correlation. However, in the literature, there are defined different measures that can be used instead of the classical ones. The first one examined here is the covariation. The measure was introduced in $[46,47]$ and for the two-dimensional symmetric $\alpha$-stable random vector with $1<\alpha<2$ denoted as $\left(Z_{1}, Z_{2}\right)$ it is defined as follows

$\operatorname{CV}\left(Z_{1}, Z_{2}\right)=\int_{S_{2}} s_{1} s_{2}^{\langle\alpha-1\rangle} \Gamma(d s)$,

where $\boldsymbol{\Gamma}$ is the spectral measure of $\left(Z_{1}, Z_{2}\right)$ and $a^{\langle p\rangle}=|a|^{p} \operatorname{sign}(a)$. The second measure commonly considered in the literature devoted to the $\alpha$ stable distribution is the codifference, and for the random vector $\left(Z_{1}, Z_{2}\right)$ is defined as follows [24]

$$
\begin{aligned}
& \mathrm{CD}\left(Z_{1}, Z_{2}\right)=\log \mathbb{E} \exp \left\{i\left(Z_{1}-Z_{2}\right)\right\}-\log \mathbb{E} \exp \left\{i Z_{1}\right\} \\
& \quad-\log \mathbb{E} \exp \left\{-i Z_{2}\right\} .
\end{aligned}
$$

Both mentioned measures differ in several properties. Namely, the covariation is defined only for the symmetric $\alpha$-stable random vectors, whereas the codifference is expressed using the characteristic function so it can be calculated for any definitely divisible distribution. Moreover, the covariation is non-symmetric in the arguments, i.e. $\mathrm{CV}\left(Z_{1}, Z_{2}\right) \neq \mathrm{CV}\left(Z_{2}, Z_{1}\right)$, on the contrary to the codifference which is symmetric in the arguments for all symmetric random vectors. For independent random variables $Z_{1}$ and $Z_{2}$, both measures take zero values. Moreover, they both reduce to the classical covariance for the Gaussian random vectors, namely

$\operatorname{Cov}\left(G_{1}, G_{2}\right)=\operatorname{CD}\left(G_{1}, G_{2}\right)=2 \mathrm{CV}\left(G_{1}, G_{2}\right)$

where $\left(G_{1}, G_{2}\right)$ is Gaussian and $\operatorname{Cov}\left(Z_{1}, Z_{2}\right)$ denotes the covariance.

The above-mentioned dependence measures are applied to describe the auto-dependence in time for the one-dimensional stochastic processes $[7,12,24,50,62-69$, 
$73,77]$, but also to describe the cross-dependence in time for the components of a two-dimensional model [70-72].

For more information about the one- and two-dimensional $\alpha$-stable distributions and the corresponding dependence measures we refer the readers to [24].

\subsection{Extended bidimensional AR(1) model}

Here, we study the model presented in Sect. 2.1 with the noise defined as in Sect. 2.2. Namely, we analyse the bidimensional time-constant AR(1) model with symmetric $\alpha$-stable noise $(1<\alpha<2)$ and the triangular coefficients matrix defined as in Eqs. (5) or (4). It is important to emphasize that although the process based on $\alpha$-stable distribution is not second-order, the form of the bounded solution is the same as in Eq. (2). In [70] the authors show that the solution of the time-constant AR(1) model with symmetric $\alpha$-stable noise is bounded in the sense of the covariation norm in the space of symmetric $\alpha$-stable random variables if the elements of matrix $\boldsymbol{\Theta}^{j}$ are absolutely summable. This condition is equivalent to the case that the eigenvalues of $\boldsymbol{\Theta}$ are less than 1 in absolute value.

In Remarks 1 and 2, we present the formulas for the cross-covariation and the cross-codifference of the bidimensional time-constant $\operatorname{AR}(1)$ model with symmetric $\alpha$ stable noise and the triangular coefficients matrix. We remind here that the cross-dependence measures describe the dependence in time between the spatial components of the multidimensional model. We omit the detailed proofs since the expressions follow directly from the formulas given in Eqs. (6)-(9) and from the general expressions presented in [71] and [72], where the model with only nonzero elements of $\boldsymbol{\Theta}$ is considered.

Remark 1 Let $\{\mathbf{X}(t)\}$ be the bidimensional time-constant $\operatorname{AR}(1)$ model with symmetric $\alpha$-stable noise $\{\mathbf{Z}(t)\}$ and the triangular coefficients matrix with the bounded solution given by Eq. (2). For $t \in \mathbb{Z}$ and $h \in \mathbb{N}_{0}$ the cross-covariation is given below.

1. For the lower-triangular coefficients matrix given in Eq. (4) and

(a) $\Theta_{11} \neq \Theta_{22}$, we have:

$$
\begin{aligned}
& \operatorname{CV}\left(X_{1}(t), X_{2}(t-h)\right)=\left(\frac{1}{\Theta_{22}-\Theta_{11}}\right)^{\langle\alpha-1\rangle} \\
& \sum_{j=0}^{+\infty} \int_{S_{2}}\left(\Theta_{22}^{j} \Theta_{21} s_{1}+\Theta_{22}^{j+1} s_{2}-\Theta_{22}^{j} \Theta_{11} s_{2}\right. \\
& \left.-\Theta_{11}^{j} \Theta_{21} s_{1}\right)^{\langle\alpha-1\rangle} \Theta_{11}^{j+h} s_{1} \Gamma(d s)
\end{aligned}
$$

$$
\begin{aligned}
& \operatorname{CV}\left(X_{1}(t), X_{2}(t+h)\right)=\left(\frac{1}{\Theta_{22}-\Theta_{11}}\right)^{\langle\alpha-1\rangle} \\
& \sum_{j=0}^{+\infty} \int_{S_{2}}\left(\Theta_{22}^{h}\left(\Theta_{22}^{j} \Theta_{21} s_{1}+\Theta_{22}^{j+1} s_{2}-\Theta_{22}^{j} \Theta_{11} s_{2}\right)\right. \\
& \left.-\Theta_{11}^{j+h} \Theta_{21} s_{1}\right)^{\langle\alpha-1\rangle} \Theta_{11}^{j} s_{1} \Gamma(d s)
\end{aligned}
$$

(b) $\Theta_{11}=\Theta_{22}=\Theta$, we have:

$$
\begin{aligned}
\operatorname{CV} & \left(X_{1}(t), X_{2}(t-h)\right) \\
= & \sum_{j=0}^{+\infty} \int_{S_{2}}\left(j \Theta^{j-1} \Theta_{21} s_{1}+\Theta^{j} s_{2}\right)^{\langle\alpha-1\rangle} \Theta^{j+h} s_{1} \Gamma(d s)
\end{aligned}
$$

$$
\begin{aligned}
\mathrm{CV} & \left(X_{1}(t), X_{2}(t+h)\right) \\
= & \sum_{j=0}^{+\infty} \int_{S_{2}}\left(\Theta^{h}\left(j \Theta^{j-1} \Theta_{21} s_{1}+\Theta^{j} s_{2}\right)\right. \\
& \left.+h \Theta^{j+h-1} \Theta_{21} s_{1}\right)^{\langle\alpha-1\rangle} \Theta^{j} s_{1} \Gamma(d s) .
\end{aligned}
$$

2. For the upper-triangular coefficients matrix given in Eq. (5) and

(a) $\Theta_{11} \neq \Theta_{22}$, we have:

$$
\begin{aligned}
& \operatorname{CV}\left(X_{1}(t), X_{2}(t-h)\right)=\frac{1}{\Theta_{22}-\Theta_{11}} \\
& \sum_{j=0}^{+\infty} \int_{S_{2}}\left(\Theta_{22}^{j} s_{2}\right)^{\langle\alpha-1\rangle}\left(\Theta _ { 1 1 } ^ { h } \left(\Theta_{11}^{j} \Theta_{22} s_{1}-\Theta_{11}^{j+1} s_{1}\right.\right. \\
& \left.\left.-\Theta_{11}^{j} \Theta_{12} s_{2}\right)+\Theta_{22}^{j+h} \Theta_{12} s_{2}\right) \Gamma(d s) \\
& \operatorname{CV}\left(X_{1}(t), X_{2}(t+h)\right)=\frac{1}{\Theta_{22}-\Theta_{11}} \\
& \sum_{j=0}^{+\infty} \int_{S_{2}}\left(\Theta_{22}^{j+h} s_{2}\right)^{\langle\alpha-1\rangle}\left(\Theta_{11}^{j} \Theta_{22} s_{1}-\Theta_{11}^{j+1} s_{1}+\Theta_{22}^{j} \Theta_{12} s_{2}\right. \\
& \left.-\Theta_{11}^{j} \Theta_{12} s_{2}\right) \Gamma(d s)
\end{aligned}
$$

(b) $\Theta_{11}=\Theta_{22}=\Theta$, we have:

$$
\begin{aligned}
\operatorname{CV} & \left(X_{1}(t), X_{2}(t-h)\right) \\
= & \sum_{j=0}^{+\infty} \int_{S_{2}}\left(\Theta^{j} s_{2}\right)^{\langle\alpha-1\rangle}\left(\Theta^{h}\left(\Theta^{j} s_{1}+j \Theta^{j-1} \Theta_{12} s_{2}\right)\right. \\
+ & \left.h \Theta^{j+h-1} \Theta_{12} s_{2}\right) \Gamma(d s) \\
\operatorname{CV} & \left(X_{1}(t), X_{2}(t+h)\right) \\
= & \sum_{j=0}^{+\infty} \int_{S_{2}}\left(\Theta^{j+h} s_{2}\right)^{\langle\alpha-1\rangle}\left(\Theta^{j} s_{1}+j \Theta^{j-1} \Theta_{12} s_{2}\right) \Gamma(d s) .
\end{aligned}
$$


Remark 2 Let $\{\mathbf{X}(t)\}$ be the bidimensional time-constant AR(1) model with symmetric $\alpha$-stable noise $\{\mathbf{Z}(t)\}$ and the triangular coefficients matrix with the bounded solution given by Eq. (2). For $t \in \mathbb{Z}$ and $h \in \mathbb{N}_{0}$, the cross-codifference is given as follows:

1. For the lower-triangular coefficients matrix given in Eq. (4) and

(a) $\Theta_{11} \neq \Theta_{22}$, we have:

$$
\begin{aligned}
\mathrm{CD}( & \left.X_{1}(t), X_{2}(t-h)\right) \\
= & \left|\frac{1}{\Theta_{22}-\Theta_{11}}\right| \sum_{j=0}^{\alpha+\infty} \int_{S_{2}}\left(\left|\Theta_{11}^{j+h}\left(\Theta_{22}-\Theta_{11}\right) s_{1}\right|^{\alpha}\right. \\
& +\left|\Theta_{22}^{j} \Theta_{21} s_{1}+\Theta_{22}^{j+1} s_{2}-\Theta_{22}^{j} \Theta_{11} s_{2}-\Theta_{11}^{j} \Theta_{21} s_{1}\right|^{\alpha} \\
& -\mid \Theta_{11}^{j+h}\left(\Theta_{22}-\Theta_{11}\right) s_{1}-\Theta_{22}^{j} \Theta_{21} s_{1}-\Theta_{22}^{j+1} s_{2} \\
+ & \left.\Theta_{22}^{j} \Theta_{11} s_{2}+\left.\Theta_{11}^{j} \Theta_{21} s_{1}\right|^{\alpha}\right) \Gamma(d s)
\end{aligned}
$$

$$
\begin{aligned}
\mathrm{CD}( & \left.X_{1}(t), X_{2}(t+h)\right) \\
= & \left|\frac{1}{\Theta_{22}-\Theta_{11}}\right| \sum_{j=0}^{\alpha+\infty} \int_{S_{2}}\left(\left|\Theta_{11}^{j}\left(\Theta_{22}-\Theta_{11}\right) s_{1}\right|^{\alpha}\right. \\
& +\left|\Theta_{22}^{h}\left(\Theta_{22}^{j} \Theta_{21} s_{1}+\Theta_{22}^{j+1} s_{2}-\Theta_{22}^{j} \Theta_{11} s_{2}\right)-\Theta_{11}^{j+h} \Theta_{21} s_{1}\right|^{\alpha} \\
& -\mid \Theta_{11}^{j}\left(\Theta_{22}-\Theta_{11}\right) s_{1}-\Theta_{22}^{h}\left(\Theta_{22}^{j} \Theta_{21} s_{1}+\Theta_{22}^{j+1} s_{2}\right. \\
- & \left.\left.\Theta_{22}^{j} \Theta_{11} s_{2}\right)+\left.\Theta_{11}^{j+h} \Theta_{21} s_{1}\right|^{\alpha}\right) \Gamma(d s)
\end{aligned}
$$

(b) $\Theta_{11}=\Theta_{22}=\Theta$, we have:

$$
\begin{aligned}
\mathrm{CD} & \left(X_{1}(t), X_{2}(t-h)\right. \\
& =\sum_{j=0}^{+\infty} \int_{S_{2}}\left(\left|\Theta^{j+h} s_{1}\right|^{\alpha}+\left|j \Theta^{j-1} \Theta_{21} s_{1}+\Theta^{j} s_{2}\right|^{\alpha}\right. \\
& -\mid \Theta^{j+h} s_{1}-j \Theta^{j-1} \Theta_{21} s_{1} . \\
& \left.\left.-\left.\Theta^{j} s_{2}\right|^{\alpha}\right) \Gamma(d s)\right)
\end{aligned}
$$

$$
\begin{aligned}
\mathrm{CD} & \left(X_{1}(t), X_{2}(t+h)\right. \\
& =\sum_{j=0}^{+\infty} \int_{S_{2}}\left(\left|\Theta^{j} s_{1}\right|^{\alpha}+\mid \Theta^{h}\left(j \Theta^{j-1} \Theta_{21} s_{1}+\Theta^{j} s_{2}\right)\right. \\
& +\left.h \Theta^{j+h-1} \Theta_{21} s_{1}\right|^{\alpha} \\
& -\mid \Theta^{j} s_{1}-\Theta^{h}\left(j \Theta^{j-1} \Theta_{21} s_{1}+\Theta^{j} s_{2}\right) \\
& \left.\left.-\left.h \Theta^{j+h-1} \Theta_{21} s_{1}\right|^{\alpha}\right) \Gamma(d s)\right)
\end{aligned}
$$

2. For the upper-triangular coefficients matrix given in Eq. (5) and

(a) $\Theta_{11} \neq \Theta_{22}$, we have:

$$
\begin{aligned}
\mathrm{CD} & \left.X_{1}(t), X_{2}(t-h)\right) \\
= & \left|\frac{1}{\Theta_{22}-\Theta_{11}}\right| \sum_{j=0}^{\alpha+\infty} \int_{S_{2}}\left(\left|\Theta_{22}^{j}\left(\Theta_{22}-\Theta_{11}\right) s_{2}\right|^{\alpha}\right. \\
& +\mid \Theta_{11}^{h}\left(\Theta_{11}^{j} \Theta_{22} s_{1}-\Theta_{11}^{j+1} s_{1}-\Theta_{11}^{j} \Theta_{12} s_{2}\right) \\
& +\left.\Theta_{22}^{j+h} \Theta_{12} s_{2}\right|^{\alpha} \\
& -\mid \Theta_{22}^{j}\left(\Theta_{22}-\Theta_{11}\right) s_{2}-\Theta_{11}^{h}\left(\Theta_{11}^{j} \Theta_{22} s_{1}-\Theta_{11}^{j+1} s_{1}\right. \\
- & \left.\left.\Theta_{11}^{j} \Theta_{12} s_{2}\right)-\left.\Theta_{22}^{j+h} \Theta_{12} s_{2}\right|^{\alpha}\right) \Gamma(d s) \\
\mathrm{CD} & \left.X_{1}(t), X_{2}(t+h)\right) \\
= & \left|\frac{1}{\Theta_{22}-\Theta_{11}}\right|^{\alpha+\infty} \sum_{j=0} \int_{S_{2}}\left(\left|\Theta_{22}^{j+h}\left(\Theta_{22}-\Theta_{11}\right) s_{2}\right|^{\alpha}\right. \\
& +\left|\Theta_{11}^{j} \Theta_{22} s_{1}-\Theta_{11}^{j+1} s_{1}+\Theta_{22}^{j} \Theta_{12} s_{2}-\Theta_{11}^{j} \Theta_{12} s_{2}\right|^{\alpha} \\
& -\mid \Theta_{22}^{j+h}\left(\Theta_{22}-\Theta_{11}\right) s_{2}-\Theta_{11}^{j} \Theta_{22} s_{1}+\Theta_{11}^{j+1} s_{1} \\
- & \left.\Theta_{22}^{j} \Theta_{12} s_{2}+\left.\Theta_{11}^{j} \Theta_{12} s_{2}\right|^{\alpha}\right) \Gamma(d s
\end{aligned}
$$

(b) $\Theta_{11}=\Theta_{22}=\Theta$, we have:

$$
\begin{aligned}
\mathrm{CD} & \left(X_{1}(t), X_{2}(t-h)\right. \\
& =\sum_{j=0}^{+\infty} \int_{S_{2}}\left(\left|\Theta^{j} s_{2}\right|^{\alpha}+\mid \Theta^{h}\left(\Theta^{j} s_{1}+j \Theta^{j-1} \Theta_{12} s_{2}\right)\right. \\
& +\left.h \Theta^{j+h-1} \Theta_{12} s_{2}\right|^{\alpha} \\
& -\mid \Theta^{j} s_{2}-\Theta^{h}\left(\Theta^{j} s_{1}+j \Theta^{j-1} \Theta_{12} s_{2}\right) \\
& \left.\left.-\left.h \Theta^{j+h-1} \Theta_{12} s_{2}\right|^{\alpha}\right) \Gamma(d s)\right)
\end{aligned}
$$




$$
\begin{aligned}
\mathrm{CD} & \left(X_{1}(t), X_{2}(t+h)\right. \\
& =\sum_{j=0}^{+\infty} \int_{S_{2}}\left(\left|\Theta^{j+h} s_{2}\right|^{\alpha}+\left|\Theta^{j} s_{1}+j \Theta^{j-1} \Theta_{12} s_{2}\right|^{\alpha}\right. \\
& \left.\left.-\left|\Theta^{j+h} s_{2}-\Theta^{j} s_{1}-j \Theta^{j-1} \Theta_{12} s_{2}\right|^{\alpha}\right) \boldsymbol{\Gamma}(d s)\right)
\end{aligned}
$$

\section{Asymptotics of cross-dependence measures}

In this section, we examine the asymptotic behaviour of the cross-dependence measures presented in Section 2.3. As one can notice, the functions given in Remarks 1-2 do not asymptotic equivalence of the functions $f(h)$ and $g(h)$ for $h \rightarrow+\infty$, i.e.

$f(h) \sim g(h)$ for $h \rightarrow+\infty \Longleftrightarrow \lim _{h \rightarrow+\infty} \frac{f(h)}{g(h)}=1$.

To facilitate the writing of the formulas in Lemmas 1-2, we introduce the cases specified in Table 1.

Lemma 1 Let $\{\mathbf{X}(t)\}$ be the bidimensional time-constant $A R(1)$ model with symmetric $\alpha$-stable noise $\{\mathbf{Z}(t)\}$ and the triangular coefficients matrix with the bounded solution given by Eq. (2). Then, for $t \in \mathbb{Z}$ and $h \in \mathbb{N}_{0}$ the crosscovariation takes the form presented below.

$$
\begin{aligned}
& \operatorname{CV}\left(X_{1}(t), X_{2}(t-h)\right) \\
& \left\{\begin{array}{lr}
=\frac{\Theta_{11}^{h}}{\left(\Theta_{22}-\Theta_{11}\right)^{\langle\alpha-1\rangle}} & \text { CASE A, B, D, E, } \\
\sum_{j=0}^{+\infty} \int_{S_{2}}\left(-\Theta_{11}^{j} \Theta_{21} s_{1}+\Theta_{22}^{j}\left(-\Theta_{11} s_{2}+\Theta_{21} s_{1}+\Theta_{22} s_{2}\right)\right)^{\langle\alpha-1\rangle}\left(\Theta_{11}^{j} s_{1}\right) \Gamma(d s) \\
{[15 p t]=\Theta^{h} \sum_{j=0}^{+\infty} \int_{S_{2}}\left(j \Theta^{j-1} \Theta_{21} s_{1}+\Theta^{j} s_{2}\right)^{\langle\alpha-1\rangle}\left(\Theta^{j} s_{1}\right) \Gamma(d s)} & \text { CASE C, }
\end{array}\right.
\end{aligned}
$$

depend on $t$. They depend only on the difference between time indexes denoted by $h$. We mention here that in Lemmas $1-2$ by the asymptotic behaviour we mean the
1. For the lower-triangular coefficients matrix given in Equation (4), we have:

2. For the upper-triangular coefficients matrix given in Eq. (5), we have:

$\mathrm{CV}\left(X_{1}(t), X_{2}(t+h)\right)$

$$
\left\{\begin{array}{cc}
\sim \frac{\Theta_{11}^{h}\langle\alpha-1\rangle}{\left(\Theta_{22}-\Theta_{11}\right)^{\langle\alpha-1\rangle}} \sum_{j=0}^{+\infty} \int_{S_{2}}\left(-\Theta_{11}^{j} \Theta_{21} s_{1}\right)^{\langle\alpha-1\rangle}\left(\Theta_{11}^{j} s_{1}\right) \Gamma(d s) & \text { CASE A, } \\
{[10 p t] \sim \frac{\Theta_{22}^{h}\langle\alpha-1\rangle}{\left(\Theta_{22}-\Theta_{11}\right)^{\langle\alpha-1\rangle}}} & \text { CASE B } \\
{[15 p t] \sim\left(h \Theta^{h}\right)^{\langle\alpha-1\rangle} \sum_{j=0}^{+\infty} \int_{S_{2}}\left(\Theta^{j-1} \Theta_{21} s_{1}\right)^{\langle\alpha-1\rangle}\left(\Theta^{j} s_{1}\right) \Gamma(d s)} & \text { CASE C } \\
{[10 p t]=\frac{\Theta_{11}^{h}\langle\alpha-1\rangle}{\left(\Theta_{22}-\Theta_{11}\right)^{\langle\alpha-1\rangle}}} & \text { CASE D } \\
\sum_{j=0}^{+\infty} \int_{S_{2}}\left(-\Theta_{11}^{j} \Theta_{21} s_{1}+\Theta_{22}^{j}\left(-\Theta_{11} s_{2}+\Theta_{21} s_{1}+\Theta_{22} s_{2}\right)\right)^{\langle\alpha-1\rangle}\left(\Theta_{11}^{j} s_{1}\right) \boldsymbol{\Gamma}(d s) & \text { CASE E. } \\
{[15 p t]=\frac{\Theta_{11}^{h}\langle\alpha-1\rangle}{\left(\Theta_{22}-\Theta_{11}\right)^{\langle\alpha-1\rangle}}} & \\
\sum_{j=0}^{+\infty} \int_{S_{2}}\left(-\Theta_{11}^{j} \Theta_{21} s_{1}-\Theta_{22}^{j}\left(-\Theta_{11} s_{2}+\Theta_{21} s_{1}+\Theta_{22} s_{2}\right)\right)^{\langle\alpha-1\rangle}\left(\Theta_{11}^{j} s_{1}\right) \boldsymbol{\Gamma}(d s) &
\end{array}\right.
$$




$$
\begin{aligned}
& \operatorname{CV}\left(X_{1}(t), X_{2}(t-h)\right) \\
& \left\{\begin{array}{rr}
\sim \frac{\Theta_{11}^{h}}{\Theta_{22}-\Theta_{11}} & \text { CASE A, } \\
{[10 p t] \sim \frac{\Theta_{22}^{h}}{\Theta_{22}-\Theta_{11}} \sum_{j=0}^{+\infty} \int_{S_{2}}\left(\Theta_{22}^{j} s_{2}\right)^{\langle\alpha-1\rangle}\left(\Theta_{22}^{j} \Theta_{12} s_{2}\right) \Gamma(d s)} & \\
{[15 p t] \sim h \Theta^{h} \sum_{j=0}^{+\infty} \int_{S_{2}}\left(\Theta^{j} s_{2}\right)^{\langle\alpha-1\rangle}\left(\Theta^{j-1} \Theta_{12} s_{2}\right) \boldsymbol{\Gamma}(d s)} & \text { CASE B }, \\
{[10 p t]=\frac{\Theta_{11}^{h}}{\Theta_{22}-\Theta_{11}}} & \text { CASE C, } \\
\sum_{j=0}^{+\infty} \int_{S_{2}}\left(\Theta_{22}^{j} s_{2}\right)^{\langle\alpha-1\rangle}\left(\Theta_{11}^{j} \Theta_{22} s_{1}-\Theta_{11}^{j+1} s_{1}-\Theta_{11}^{j} \Theta_{12} s_{2}+\Theta_{22}^{j} \Theta_{12} s_{2}\right) \boldsymbol{\Gamma}(d s) & \text { CASE D, } \\
{[15 p t]=\frac{\Theta_{11}^{h}}{\Theta_{22}-\Theta_{11}}} & \text { CASE E, } \\
\sum_{j=0}^{+\infty} \int_{S_{2}}\left(\Theta_{22}^{j} s_{2}\right)^{\langle\alpha-1\rangle}\left(\Theta_{11}^{j} \Theta_{22} s_{1}-\Theta_{11}^{j+1} s_{1}-\Theta_{11}^{j} \Theta_{12} s_{2}-\Theta_{22}^{j} \Theta_{12} s_{2}\right) \boldsymbol{\Gamma}(d s) &
\end{array}\right.
\end{aligned}
$$

The symbol " $\sim$ " denotes the asymptotic behaviour, according to the condition given in Eq. (30), and the symbol " =" denotes the equality.

Proof A sketch of the proof for a sample case is presented in "Appendix 1".
Lemma 2 Let $\{\mathbf{X}(t)\}$ be the bidimensional time-constant $A R(1)$ model with symmetric $\alpha$-stable noise $\{\mathbf{Z}(t)\}$ and the triangular coefficients matrix with the bounded solution given by Eq. (2). Then, for $t \in \mathbb{Z}$ and $h \in \mathbb{N}_{0}$ the crosscodifference takes the form presented below.

1. For the lower-triangular coefficients matrix given in Eq. (4), we have:

$$
\begin{aligned}
& \mathrm{CV}\left(X_{1}(t), X_{2}(t+h)\right) \\
& \left\{\begin{array}{lr}
=\frac{\Theta_{22}^{h\langle\alpha-1\rangle}}{\Theta_{22}-\Theta_{11}} & \text { CASE A, B, D, E, } \\
\left.\sum_{j=0}^{+\infty} \int_{S_{2}}\left(\Theta_{22}^{j} s_{2}\right)^{\langle\alpha-1\rangle}\left(\Theta_{11}^{j}\left(\Theta_{22} s_{1}-\Theta_{11} s_{1}-\Theta_{12} s_{2}\right)+\Theta_{22}^{j} \Theta_{12} s_{2}\right)\right) \Gamma(d s) & \text { CASE C. }
\end{array}\right.
\end{aligned}
$$

$$
\begin{aligned}
& \mathrm{CD}\left(X_{1}(t), X_{2}(t-h)\right) \\
& \left\{\begin{array}{cc}
\sim \frac{\alpha \Theta_{11}^{h}}{\left(\Theta_{22}-\Theta_{11}\right)^{\langle\alpha-1\rangle}} & \text { CASE A, B, D, E, } \\
\sum_{j=0}^{+\infty} \int_{S_{2}}\left(-\Theta_{11}^{j} \Theta_{21} s_{1}+\Theta_{22}^{j}\left(-\Theta_{11} s_{2}+\Theta_{21} s_{1}+\Theta_{22} s_{2}\right)\right)^{\langle\alpha-1\rangle}\left(\Theta_{11}^{j} s_{1}\right) \Gamma(d s) & \text { CASE C },
\end{array}\right.
\end{aligned}
$$


$\mathrm{CD}\left(X_{1}(t), X_{2}(t+h)\right)$

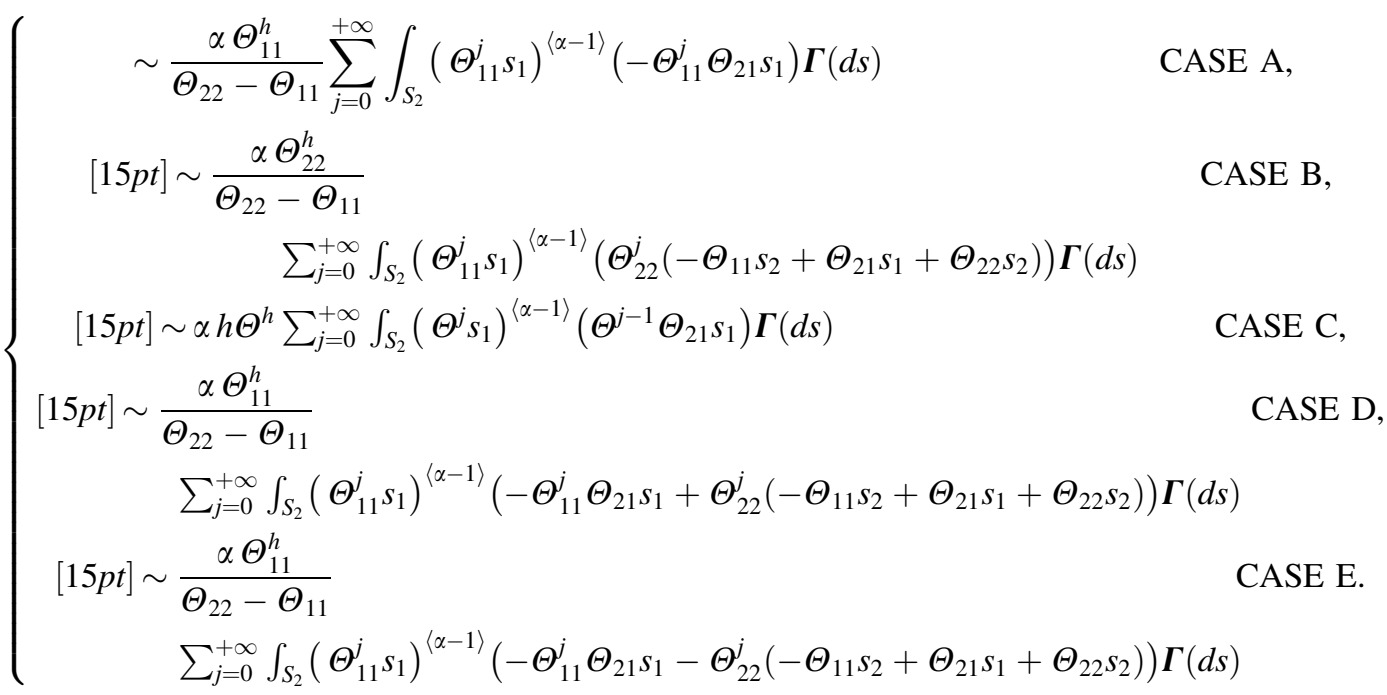

2. For the upper-triangular coefficients matrix given in Eq. (5), we have:
The symbol " " denotes the asymptotic behaviour according to the condition given in Eq. (30).

$$
\begin{aligned}
& \mathrm{CD}\left(X_{1}(t), X_{2}(t-h)\right) \\
& \left\{\begin{array}{rr}
\sim \frac{\alpha \Theta_{11}^{h}}{\Theta_{22}-\Theta_{11}} & \text { CASE A, } \\
{[10 p t] \sim \frac{\alpha \Theta_{22}^{h}}{\Theta_{22}-\Theta_{11}} \sum_{j=0}^{+\infty} \int_{S_{2}}\left(\Theta_{22}^{j} s_{2}\right)^{\langle\alpha-1\rangle}\left(\Theta_{22}^{j} \Theta_{12} s_{2}\right) \Gamma(d s)} & \text { CASE B, } \\
{[15 p t] \sim \alpha h \Theta^{h} \sum_{j=0}^{+\infty} \int_{S_{2}}\left(\Theta^{j} s_{2}\right)^{\langle\alpha-1\rangle}\left(\Theta^{j-1} \Theta_{12} s_{2}\right) \Gamma(d s)} & \text { CASE C }, \\
{[10 p t] \sim \frac{\alpha \Theta_{11}^{h}}{\Theta_{22}-\Theta_{11}}} & \text { CASE D, } \\
{[15 p t] \sim \frac{\alpha \Theta_{11}^{h}}{\Theta_{22}^{+\infty}-\Theta_{11} \int_{S_{2}}}\left(\Theta_{22}^{j} s_{2}\right)^{\langle\alpha-1\rangle}\left(\Theta_{11}^{j} \Theta_{22} s_{1}-\Theta_{11}^{j+1} s_{1}-\Theta_{11}^{j} \Theta_{12} s_{2}+\Theta_{22}^{j} \Theta_{12} s_{2}\right) \Gamma(d s)} & \text { CASE E }
\end{array}\right.
\end{aligned}
$$




$$
\begin{aligned}
& \operatorname{CD}\left(X_{1}(t), X_{2}(t+h)\right) \\
& \begin{cases}\sim \frac{\Theta_{22}^{h}}{\left(\Theta_{22}-\Theta 11\right)^{\langle\alpha-1\rangle}} & \text { CASE A, B, D, E, } \\
\left.\sum_{j=0}^{+\infty} \int_{S_{2}}\left(\Theta_{11}^{j}\left(\Theta_{22} s_{2}-\Theta_{11} s_{1}-\Theta_{12} s_{2}\right)+\Theta_{22}^{j} \Theta_{12} s_{2}\right)\right)^{\langle\alpha-1\rangle}\left(\Theta_{22}^{j} s_{2}\right) \Gamma(d s) & \text { CASE C. }\end{cases}
\end{aligned}
$$

Table 1 The considered cases for the asymptotic behaviour of the cross-dependence measures presented in Remarks 1-2

\begin{tabular}{ll}
\hline CASE A & $\left|\Theta_{11}\right|>\left|\Theta_{22}\right|$ \\
CASE B & $\left|\Theta_{11}\right|<\left|\Theta_{22}\right|$ \\
CASE C & $\Theta_{11}=\Theta_{22}$ \\
CASE D & $\Theta_{11}=-\Theta_{22}$ and $h$ even \\
CASE E & $\Theta_{11}=-\Theta_{22}$ and $h$ odd \\
\hline
\end{tabular}

Proof A sketch of the proof for a sample case is presented in "Appendix 2".

The results presented in Lemmas 1-2 show that the behaviour of cross-dependence measures for $h \rightarrow+\infty$ depends on which spatial component of the bidimensional time series is taken with a greater time index. If the argument with a greater time index corresponds to the "simplified" component-the one reduced to one-dimensional AR(1) model - then, the obtained formula is based always on the same term, $\Theta_{11}^{h}$ for lower-triangular coefficients matrix or $\Theta_{22}^{h}$ for upper-triangular coefficients matrix, regardless on the relation between $\Theta_{11}$ and $\Theta_{22}$. We can say that in this case, in the formulas for the crossdependence measures, the coefficient corresponding to the simplified model "dominates" the other coefficient located on the main diagonal. Otherwise, if the argument with a greater time index corresponds to the "non-simplified" component, we observe different kinds of behaviour depending on the relation between $\Theta_{11}$ and $\Theta_{22}$.

Based on the expressions presented in Lemmas 1-2, we can formulate the theorem regarding the asymptotic relation between the cross-codifference and the crosscovariation.

Theorem 1 Let $\{\mathbf{X}(t)\}$ be the bidimensional time-constant $A R(1)$ model with symmetric $\alpha$-stable noise $\{\mathbf{Z}(t)\}$ and the triangular coefficients matrix with the bounded solution given by Eq. (2). Then, for each $t \in \mathbb{Z}$ the following limits hold $\lim _{h \rightarrow+\infty} \frac{\operatorname{CD}\left(X_{1}(t), X_{2}(t-h)\right)}{\operatorname{CV}\left(X_{1}(t), X_{2}(t-h)\right)}=\alpha$,

and

$$
\lim _{h \rightarrow+\infty} \frac{\operatorname{CD}\left(X_{1}(t), X_{2}(t+h)\right)}{\operatorname{CV}\left(X_{1}(t), X_{2}(t+h)\right)}=0,
$$

assuming that the cross-covariation in the denominators are nonzero for all values of $t \in \mathbb{Z}$ and $h \in \mathbb{N}_{0}$.

Proof The proof directly follows from the formulas presented in Lemmas 1-2.

According to Theorem 1, in the case when the time index of the second argument is less than the time index of the first argument, the cross-codifference and the crosscovariation are asymptotically proportional with the index of proportionality equal to $\alpha$. We mention here that the analogous theorem is true also for the bidimensional timeconstant $\operatorname{AR}(1)$ model with $\alpha$-stable noise and the nontriangular matrix, see [72].

\section{Illustration}

In this section, we illustrate the results presented in Theorem 1 for sample bidimensional time-constant $\operatorname{AR}(1)$ model with $\alpha$-stable noise and the triangular matrix. We take a particular distribution of $\alpha$-stable noise $\mathbf{Z}(t)$ by assuming a discrete spectral measure of the following form

$$
\begin{aligned}
\Gamma(\cdot)= & \gamma_{1} \delta\left(\left(\frac{\sqrt{2}}{2}, \frac{\sqrt{2}}{2}\right)\right)+\gamma_{1} \delta\left(\left(-\frac{\sqrt{2}}{2},-\frac{\sqrt{2}}{2}\right)\right) \\
& +\gamma_{2} \delta\left(\left(-\frac{\sqrt{2}}{2}, \frac{\sqrt{2}}{2}\right)\right) \\
& +\gamma_{2} \delta\left(\left(\frac{\sqrt{2}}{2},-\frac{\sqrt{2}}{2}\right)\right),
\end{aligned}
$$


where $\gamma_{1}=0.1$ and $\gamma_{2}=0.3$ are the weights attributed to the point masses localized on the unit circle $S_{2}$. We mention here that since the weights of the antipodal points are equal, the random vector $\mathbf{Z}(t)$ is symmetric. Moreover, the noise components are dependent, since the mass points there are not localized on the intersection of $S_{2}$ with the coordinate system axes [24].

In Figs. 1, 2, 3 and 4, we present the theoretical values taken by the cross-codifference and cross-covariation ratios for two sample triangular matrices. Figures 1 and 2 correspond to the case of

$\boldsymbol{\Theta}^{(1)}=\left[\begin{array}{cc}0.6 & 0 \\ 0.8 & -0.2\end{array}\right]$

and Figs. 3 and 4 present the results corresponding to

$\boldsymbol{\Theta}^{(2)}=\left[\begin{array}{cc}0.6 & 0.8 \\ 0 & -0.2\end{array}\right]$.

In Figs. 1 and 3, the values are additionally divided by the stability index. One can observe that the obtained results illustrate the theorem presented in Sect. 3. Namely, as the value of $h$ is getting bigger the values of the examined ratios are getting closer to 1 or 0 , depending on whether the time index of the second argument is smaller or bigger than the time index of the first argument.

\section{Conclusions}

In this paper, we examine the dependence in time between the spatial components of the bidimensional time-constant AR(1) model with $\alpha$-stable noise. Similar considerations were presented in the author's previous papers, see [70-72]. However, here we study the case of the triangular coefficients matrix resulting in the fact that one of the spatial components simplifies to the one-dimensional AR(1) model.

For the considered model, the formulas for the crosscodifference and the cross-covariation are presented, that

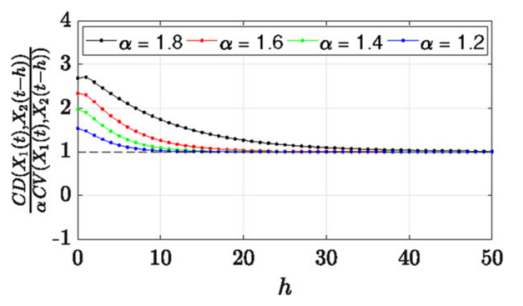

Fig. 1 The theoretical value of the cross-codifference and crosscovariation ratio for sample bidimensional time-constant $\mathrm{AR}(1)$ model with $\alpha$-stable noise and lower-triangular coefficients matrix

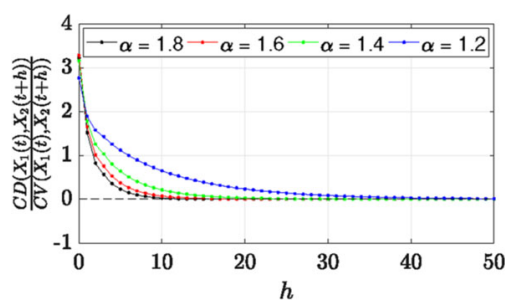

Fig. 2 The theoretical value of the cross-codifference and crosscovariation ratio for sample bidimensional time-constant $\mathrm{AR}(1)$ model with $\alpha$-stable noise and lower-triangular coefficients matrix

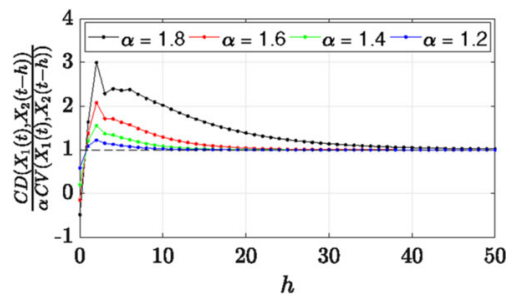

Fig. 3 The theoretical value of the cross-codifference and crosscovariation ratio for sample bidimensional time-constant $\operatorname{AR}(1)$ model with $\alpha$-stable noise and upper-triangular coefficients matrix

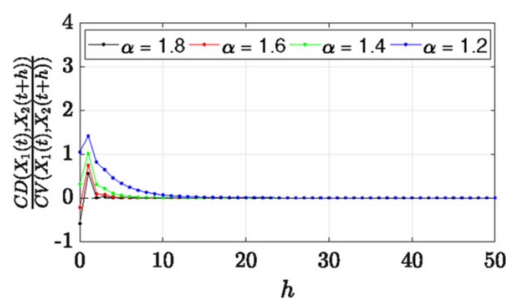

Fig. 4 The theoretical value of the cross-codifference and crosscovariation ratio for sample bidimensional time-constant $\operatorname{AR}(1)$ model with $\alpha$-stable noise and upper-triangular coefficients matrix

result from the general expressions given in [71, 72]. Then, we examine the asymptotic behaviour of the cross-dependence measures which is shown to depend on which spatial component is taken with a greater time index. Based on the asymptotic formulas, we formulate a theorem regarding the asymptotic relation between two measures of cross-dependence which is analogous to the theorem obtained for the model with the non-triangular matrix. In the case when the time index of the second argument is smaller than the time index of the first argument the measures are asymptotically proportional with the index of proportionality equal to the stability parameter.

The results presented in this paper, together with the ones provided in [72], allow us to fully describe the asymptotics of the examined cross-dependence measures for the bidimensional time-constant AR(1) model with $\alpha$ stable noise in the case of both triangular or non-triangular coefficients matrix. 
Open Access This article is licensed under a Creative Commons Attribution 4.0 International License, which permits use, sharing, adaptation, distribution and reproduction in any medium or format, as long as you give appropriate credit to the original author(s) and the source, provide a link to the Creative Commons licence, and indicate if changes were made. The images or other third party material in this article are included in the article's Creative Commons licence, unless indicated otherwise in a credit line to the material. If material is not included in the article's Creative Commons licence and your intended use is not permitted by statutory regulation or exceeds the permitted use, you will need to obtain permission directly from the copyright holder. To view a copy of this licence, visit http://creativecommons.org/licenses/by/4.0/.

\section{Appendix 1: Proof of Lemma 1}

Proof For some expressions presented in Lemma 1, we have the exact formulas, which is denoted by "=". For the others, written with the symbol " ", the asymptotic behaviour is examined in a very similar manner. Below, we present the proof only for one sample case included in Lemma 1.

Let us examine the asymptotic behaviour of $\mathrm{CV}\left(X_{1}(t), X_{2}(t+h)\right)$ given in Eq. (15) for the lowertriangular coefficients matrix. Moreover, let us consider CASE A, i.e. $\left|\Theta_{11}\right|>\left|\Theta_{22}\right|$. The examined function can be written in the following form

$\mathrm{CV}\left(X_{1}(t), X_{2}(t+h)\right)=c \sum_{j=0}^{+\infty} \int_{S_{2}}\left(c_{1} \Theta_{11}^{h}+c_{2} \Theta_{22}^{h}\right)^{\langle\alpha-1\rangle} c_{3} \Gamma(d s)$

where

$$
\begin{aligned}
& c=\left(\left(\Theta_{22}-\Theta_{11}\right)^{-1}\right)^{\langle\alpha-1\rangle}, \\
& c_{2}=\Theta_{22}^{j} \Theta_{21} s_{1}+\Theta_{22}^{j+1} s_{2}-\Theta_{22}^{j} \Theta_{11} s_{2},
\end{aligned}
$$

The proof is divided into two main steps. Namely, we show that two following equalities hold

(i)

$$
\begin{aligned}
& \lim _{h \rightarrow+\infty} \sum_{j=0}^{+\infty} \int_{S_{2}} \frac{\left(c_{1} \Theta_{11}^{h}+c_{2} \Theta_{22}^{h}\right)^{\langle\alpha-1\rangle} c_{3}}{\left(\Theta_{11}^{h}\right)^{\langle\alpha-1\rangle}} \boldsymbol{\Gamma}(d s) \\
& =\sum_{j=0}^{+\infty} \lim _{h \rightarrow+\infty} \int_{S_{2}} \frac{\left(c_{1} \Theta_{11}^{h}+c_{2} \Theta_{22}^{h}\right)^{\langle\alpha-1\rangle} c_{3}}{\left(\Theta_{11}^{h}\right)^{\langle\alpha-1\rangle}} \boldsymbol{\Gamma}(d s),
\end{aligned}
$$

$$
\begin{aligned}
& \lim _{h \rightarrow+\infty} \int_{S_{2}} \frac{\left(c_{1} \Theta_{11}^{h}+c_{2} \Theta_{22}^{h}\right)^{\langle\alpha-1\rangle} c_{3}}{\left(\Theta_{11}^{h}\right)^{\langle\alpha-1\rangle}} \boldsymbol{\Gamma}(d s) \\
& =\int_{S_{2}} \lim _{h \rightarrow+\infty} \frac{\left(c_{1} \Theta_{11}^{h}+c_{2} \Theta_{22}^{h}\right)^{\langle\alpha-1\rangle} c_{3}}{\left(\Theta_{11}^{h}\right)^{\langle\alpha-1\rangle}} \boldsymbol{\Gamma}(d s) .
\end{aligned}
$$

To show that the above equalities are true, one may use the dominated convergence theorem. Namely, since for all $m, n \in \mathbb{R}$ and $1<\alpha<2$

$|m+n|^{\alpha-1} \leq|m|^{\alpha-1}+|n|^{\alpha-1}$

and therefore,

$$
\begin{aligned}
& \left|\int_{S_{2}} \frac{\left(c_{1} \Theta_{11}^{h}+c_{2} \Theta_{22}^{h}\right)^{\langle\alpha-1\rangle} c_{3}}{\left(\Theta_{11}^{h}\right)^{\langle\alpha-1\rangle}} \boldsymbol{\Gamma}(d s)\right| \\
& \quad \leq \int_{S_{2}}\left|c_{1}\right|^{\alpha-1}\left|c_{3}\right| \boldsymbol{\Gamma}(d s)+\int_{S_{2}}\left|c_{2}\right|^{\alpha-1}\left|c_{3}\right| \boldsymbol{\Gamma}(d s),
\end{aligned}
$$

the infinite sum under the limit on the left-hand side of Eq. (47) converges uniformly if the following conditions are satisfied

$$
\begin{aligned}
& \sum_{j=0}^{+\infty} \int_{S_{2}}\left|c_{1}\right|^{\alpha-1}\left|c_{3}\right| \boldsymbol{\Gamma}(d s)<+\infty \\
& \sum_{j=0}^{+\infty} \int_{S_{2}}\left|c_{2}\right|^{\alpha-1}\left|c_{3}\right| \boldsymbol{\Gamma}(d s)<+\infty
\end{aligned}
$$

which is true since the spectral measure $\Gamma$ is finite and the absolute values of $c_{1}, c_{2}$ and $c_{3}$ can by upper-bounded by $K \max \left(\left|\Theta_{11}\right|,\left|\Theta_{22}\right|\right)^{j}$, where $K$ is independent of $j$, and $\max \left(\left|\Theta_{11}\right|,\left|\Theta_{22}\right|\right)<1$. Therefore, the equality given in Eq. (47) is true. Then, since for a fixed $\mathbf{s}=\left(s_{1}, s_{2}\right) \in S_{2}$ we have that

$$
\left|\frac{\left(c_{1} \Theta_{11}^{h}+c_{2} \Theta_{22}^{h}\right)^{\langle\alpha-1\rangle} c_{3}}{\left(\Theta_{11}^{h}\right)^{\langle\alpha-1\rangle}}\right| \leq\left|c_{1}\right|^{\alpha-1}\left|c_{3}\right|+\left|c_{2}\right|^{\alpha-1}\left|c_{3}\right|
$$

and the expression on right-hand side of the above inequality is independent of $h$, Eq. (48) holds if for all $j \in \mathbb{N}$

$$
\int_{S_{2}}\left|c_{1}\right|^{\alpha-1}\left|c_{3}\right| \boldsymbol{\Gamma}(d s)<+\infty, \quad \int_{S_{2}}\left|c_{2}\right|^{\alpha-1}\left|c_{3}\right| \boldsymbol{\Gamma}(d s)<+\infty,
$$

which is true due to the same argument as for Eq. (50). Consequently, since for a fixed $\mathbf{s}=\left(s_{1}, s_{2}\right) \in S_{2}$ we have

$$
\lim _{h \rightarrow+\infty} \frac{\left(c_{1} \Theta_{11}^{h}+c_{2} \Theta_{22}^{h}\right)^{\langle\alpha-1\rangle} c_{3}}{\left(\Theta_{11}^{h}\right)^{\langle\alpha-1\rangle}}=c_{1}^{\langle\alpha-1\rangle} c_{3}
$$

finally, we obtain that 


$$
\begin{aligned}
& \lim _{h \rightarrow+\infty} \sum_{j=0}^{+\infty} \int_{S_{2}} \frac{\left(c_{1} \Theta_{11}^{h}+c_{2} \Theta_{22}^{h}\right)^{\langle\alpha-1\rangle} c_{3}}{\left(\Theta_{11}^{h}\right)^{\langle\alpha-1\rangle}} \boldsymbol{\Gamma}(d s) \\
& =\sum_{j=0}^{+\infty} \int_{S_{2}} c_{1}^{\langle\alpha-1\rangle} c_{3} \boldsymbol{\Gamma}(d s) .
\end{aligned}
$$

Therefore,

$$
\begin{gathered}
c \sum_{j=0}^{+\infty} \int_{S_{2}}\left(c_{1} \Theta_{11}^{h}+c_{2} \Theta_{22}^{h}\right)^{\langle\alpha-1\rangle} c_{3} \boldsymbol{\Gamma}(d s) \\
\sim\left(\Theta_{11}^{h}\right)^{\langle\alpha-1\rangle} c \sum_{j=0}^{+\infty} \int_{S_{2}} c_{1}^{\langle\alpha-1\rangle} c_{3} \boldsymbol{\Gamma}(d s)
\end{gathered}
$$

for $h \rightarrow+\infty$, which in line with Eq. (32) CASE A.

\section{Appendix 2: Proof of Lemma 2}

Proof Below, we present the proof only for one sample case included in Lemma 2. We mention that for the other formulas the asymptotic behaviour is examined in a very similar manner.

Let us study the asymptotic behaviour of $\mathrm{CD}\left(X_{1}(t), X_{2}(t+h)\right)$ given in Eq. (23) for the lowertriangular coefficients matrix. Moreover, let us focus on CASE A, i.e. $\left|\Theta_{11}\right|>\left|\Theta_{22}\right|$. The examined function can be written in the following form

$$
\begin{aligned}
\mathrm{CD} & \left(X_{1}(t), X_{2}(t+h)\right) \\
& =d \sum_{j=0}^{+\infty} \int_{S_{2}}\left(\left|\Theta_{11}^{h} d_{1}+\Theta_{22}^{h} d_{2}\right|^{\alpha}+\left|d_{3}\right|^{\alpha}\right. \\
& \left.-\left|d_{3}-\left(\Theta_{11}^{h} d_{1}+\Theta_{22}^{h} d_{2}\right)\right|^{\alpha}\right) \Gamma(d s)
\end{aligned}
$$

where

$$
\begin{aligned}
& d=\left|\Theta_{22}-\Theta_{11}\right|^{-\alpha} \\
& d_{2}=\left(\Theta_{22}^{j} \Theta_{21} s_{1}+\Theta_{22}^{j+1} s_{2}-\Theta_{22}^{j} \Theta_{11} s_{2}\right)
\end{aligned}
$$

Similarly to the proof of Lemma 1, here we show that two following equalities hold

(i)

$$
\begin{aligned}
& \lim _{h \rightarrow+\infty} \sum_{j=0}^{+\infty} \int_{S_{2}} \frac{\left|\Theta_{11}^{h} d_{1}+\Theta_{22}^{h} d_{2}\right|^{\alpha}+\left|d_{3}\right|^{\alpha}-\left|d_{3}-\left(\Theta_{11}^{h} d_{1}+\Theta_{22}^{h} d_{2}\right)\right|^{\alpha}}{\Theta_{11}^{h}} \boldsymbol{\Gamma}(d s) \\
& =\sum_{j=0}^{+\infty} \lim _{h \rightarrow+\infty} \int_{S_{2}} \frac{\left|\Theta_{11}^{h} d_{1}+\Theta_{22}^{h} d_{2}\right|^{\alpha}+\left|d_{3}\right|^{\alpha}-\left|d_{3}-\left(\Theta_{11}^{h} d_{1}+\Theta_{22}^{h} d_{2}\right)\right|^{\alpha}}{\Theta_{11}^{h}} \boldsymbol{\Gamma}(d s),
\end{aligned}
$$

(ii)

$$
\begin{aligned}
& \lim _{h \rightarrow+\infty} \int_{S_{2}} \frac{\left|\Theta_{11}^{h} d_{1}+\Theta_{22}^{h} d_{2}\right|^{\alpha}+\left|d_{3}\right|^{\alpha}-\left|d_{3}-\left(\Theta_{11}^{h} d_{1}+\Theta_{22}^{h} d_{2}\right)\right|^{\alpha}}{\Theta_{11}^{h}} \Gamma(d s) \\
& =\int_{S_{2}} \lim _{h \rightarrow+\infty} \frac{\left|\Theta_{11}^{h} d_{1}+\Theta_{22}^{h} d_{2}\right|^{\alpha}+\left|d_{3}\right|^{\alpha}-\left|d_{3}-\left(\Theta_{11}^{h} d_{1}+\Theta_{22}^{h} d_{2}\right)\right|^{\alpha}}{\Theta_{11}^{h}} \boldsymbol{\Gamma}(d s) .
\end{aligned}
$$

The above equalities are true due to the dominated convergence theorem. Namely, since for all $n, m \in$ $\mathbb{R}, 1<\alpha<2$ we have [65]

$$
\begin{aligned}
& \left.|| n\right|^{\alpha}+|m|^{\alpha}-\left.|n+m|^{\alpha}|\leq(\alpha+1)| n\right|^{\alpha}+\alpha|n||m|^{\alpha-1} \\
& |n+m|^{\alpha} \leq 2^{\alpha-1}\left(|n|^{\alpha}+|m|^{\alpha}\right),
\end{aligned}
$$

and therefore, for all $j \in \mathbb{N}$ we have

$$
\begin{aligned}
\left|\int_{S_{2}} \frac{\left|\Theta_{11}^{h} d_{1}+\Theta_{22}^{h} d_{2}\right|^{\alpha}+\left|d_{3}\right|^{\alpha}-\left|d_{3}-\left(\Theta_{11}^{h} d_{1}+\Theta_{22}^{h} d_{2}\right)\right|^{\alpha}}{\Theta_{11}^{h}} \boldsymbol{\Gamma}(d s)\right| \\
\leq 2^{\alpha-1}(\alpha+1)\left(\int_{S_{2}}\left|d_{1}\right|^{\alpha} \boldsymbol{\Gamma}(d s)+\int_{S_{2}}\left|d_{2}\right|^{\alpha} \boldsymbol{\Gamma}(d s)\right) \\
\quad+\alpha\left(\int_{S_{2}}\left|d_{1}\right|\left|d_{3}\right|^{\alpha-1} \boldsymbol{\Gamma}(d s)+\int_{S_{2}}\left|d_{2}\right|\left|d_{3}\right|^{\alpha-1} \boldsymbol{\Gamma}(d s)\right),
\end{aligned}
$$

the infinite sum under the limit on the left-hand side of Eq. (57) converges uniformly if the following conditions are satisfied

$$
\begin{array}{ll}
\sum_{j=0}^{+\infty} \int_{S_{2}}\left|d_{1}\right|^{\alpha} \boldsymbol{\Gamma}(d s)<+\infty, & \sum_{j=0}^{+\infty} \int_{S_{2}}\left|d_{1}\right|\left|d_{3}\right|^{\alpha-1} \boldsymbol{\Gamma}(d s)<+\infty, \\
\sum_{j=0}^{+\infty} \int_{S_{2}}\left|d_{2}\right|^{\alpha} \boldsymbol{\Gamma}(d s)<+\infty, & \sum_{j=0}^{+\infty} \int_{S_{2}}\left|d_{2}\right|\left|d_{3}\right|^{\alpha-1} \boldsymbol{\Gamma}(d s)<+\infty,
\end{array}
$$

which is always true. Therefore, the equality given in Eq. (57) hold. Then, since for a fixed $\mathbf{s}=\left(s_{1}, s_{2}\right) \in S_{2}$ we have that

$$
\begin{aligned}
& \left|\frac{\left|\Theta_{11}^{h} d_{1}+\Theta_{22}^{h} d_{2}\right|^{\alpha}+\left|d_{3}\right|^{\alpha}-\left|d_{3}-\left(\Theta_{11}^{h} d_{1}+\Theta_{22}^{h} d_{2}\right)\right|^{\alpha} \mid}{\Theta_{11}^{h}}\right| \\
& \leq 2^{\alpha-1}(\alpha+1)\left(\left|d_{1}\right|^{\alpha}+\left|d_{2}\right|^{\alpha}\right)+\alpha\left(\left|d_{1}\right|\left|d_{3}\right|^{\alpha-1}+\left|d_{2}\right|\left|d_{3}\right|^{\alpha-1}\right),
\end{aligned}
$$

and the expression on the right-hand side of the above inequality is independent of $h$, Eq. (58) holds if for all $j \in \mathbb{N}$ the following conditions hold

$$
\begin{array}{ll}
\int_{S_{2}}\left|d_{1}\right|^{\alpha} \boldsymbol{\Gamma}(d s)<+\infty, & \int_{S_{2}}\left|d_{1}\right|\left|d_{3}\right|^{\alpha-1} \boldsymbol{\Gamma}(d s)<+\infty, \\
\int_{S_{2}}\left|d_{2}\right|^{\alpha} \boldsymbol{\Gamma}(d s)<+\infty, & \int_{S_{2}}\left|d_{2}\right|\left|d_{3}\right|^{\alpha-1} \boldsymbol{\Gamma}(d s)<+\infty,
\end{array}
$$

which is true. Consequently, since for a fixed $\mathbf{s}=$ $\left(s_{1}, s_{2}\right) \in S_{2}$ and $h \rightarrow+\infty$ we have 


$$
\begin{aligned}
& \left|\Theta_{11}^{h} d_{1}+\Theta_{22}^{h} d_{2}\right|^{\alpha}+\left|d_{3}\right|^{\alpha}-\left|d_{3}-\left(\Theta_{11}^{h} d_{1}+\Theta_{22}^{h} d_{2}\right)\right|^{\alpha} \\
& \quad=\left|\Theta_{11}^{h}\left(d_{1}+\left(\Theta_{22} / \Theta_{11}\right)^{h} d_{2}\right)\right|^{\alpha}+\left|d_{3}\right|^{\alpha}-\mid d_{3} \\
& -\left.\Theta_{11}^{h}\left(d_{1}+\left(\Theta_{22} / \Theta_{11}\right)^{h} d_{2}\right)\right|^{\alpha} \\
& \sim\left|\Theta_{11}^{h}\right|^{\alpha}\left|d_{1}\right|^{\alpha}+\left|d_{3}\right|^{\alpha}-\left|d_{3}-\Theta_{11}^{h} d_{1}\right|^{\alpha}
\end{aligned}
$$

and since for $1<\alpha<2$ and $r_{1}, r_{2} \in \mathbb{R}$ the below limit holds

$$
\lim _{x \rightarrow 0} \frac{\left|r_{1} x\right|^{\alpha}+\left|r_{2}\right|^{\alpha}-\left|r_{2}-r_{1} x\right|^{\alpha}}{x}=\alpha r_{1} r_{2}^{\langle\alpha-1\rangle}
$$

and moreover, $\Theta_{11}^{h} \rightarrow 0$ as $h \rightarrow+\infty$, finally, we obtain that

$$
\lim _{h \rightarrow+\infty} \frac{\left|\Theta_{11}^{h} d_{1}\right|^{\alpha}+\left|d_{3}\right|^{\alpha}-\left|d_{3}-\Theta_{11}^{h} d_{1}\right|^{\alpha}}{\Theta_{11}^{h}}=\alpha d_{1} d_{3}^{\langle\alpha-1\rangle}
$$

for a fixed $\mathbf{s}=\left(s_{1}, s_{2}\right) \in S_{2}$. Therefore,

$$
\begin{aligned}
& \lim _{h \rightarrow+\infty} \sum_{j=0}^{+\infty} \\
& \int_{S_{2}} \frac{\left|\Theta_{11}^{h} d_{1}+\Theta_{22}^{h} d_{2}\right|^{\alpha}+\left|d_{3}\right|^{\alpha}-\left|d_{3}-\left(\Theta_{11}^{h} d_{1}+\Theta_{22}^{h} d_{2}\right)\right|^{\alpha}}{\Theta_{11}^{h}} \\
& \Gamma(d s) \\
& =\alpha \sum_{j=0}^{+\infty} \int_{S_{2}} d_{1} d_{3}^{\langle\alpha-1\rangle} \boldsymbol{\Gamma}(d s) .
\end{aligned}
$$

Finally, we have

$$
\begin{gathered}
d \sum_{j=0}^{+\infty} \int_{S_{2}}\left|\Theta_{11}^{h} d_{1}+\Theta_{22}^{h} d_{2}\right|^{\alpha}+\left|d_{3}\right|^{\alpha} \\
-\left|d_{3}-\left(\Theta_{11}^{h} d_{1}+\Theta_{22}^{h} d_{2}\right)\right|^{\alpha} \boldsymbol{\Gamma}(d s) \\
\quad \sim \alpha d \Theta_{11}^{h} \sum_{j=0}^{+\infty} \int_{S_{2}} d_{1} d_{3}^{\langle\alpha-1\rangle} \boldsymbol{\Gamma}(d s)
\end{gathered}
$$

for $h \rightarrow+\infty$, which is in line with Eq. (36) CASE A.

\section{References}

1. Yule, G.U.: On a method of investigating periodicities in disturbed series, with special reference to Wolfer's sunspot numbers. Philosophical Transactions of the Royal Society of London. Series A, Containing Papers of a Mathematical or Physical Character 226, 267-298 (1927)

2. Hamilton, J.: Time Series Analysis. Princeton University Press, Princeton (1994)

3. Lütkepohl, H.: New introduction to multiple time series analysis. Springer, Berlin (2005)

4. Brockwell, P.J., Davis, R.A.: Introduction to Time Series and Forecasting. Springer, Berlin (2016)
5. Davis, R.A., Knight, K., Liu, J.: M-estimation for autoregressions with infinite variance. Stochas. Processes Appl. 40(1), 145-180 (1992)

6. Kokoszka, P.S., Taqqu, M.S.: Infinite variance stable ARMA processes. J. Time Ser. Anal. 15(2), 203-220 (1994)

7. Nowicka, J.: Asymptotic behavior of the covariation and the codifference for ARMA models with stable innovations. Commun. Stat. Stochastic Models 13(4), 673-685 (1997)

8. Kuruoglu, E.E., Rayner, P.J.W., Fitzgerald, W.J.: Least $L_{p}$-norm estimation of autoregressive model coefficients of symmetric $\alpha$ stable processes. IEEE Signal Process. Lett. 4(7), 201-203 (1997)

9. Maymon, S., Friedmann, J., Messer, H.: On AR parameter estimation with alpha stable innovations. In: Proceedings of the IEEE Signal Processing Workshop on Higher-Order Statistics. SPWHOS '99, pp. 237-240 (1999)

10. Mikosch, T., Gadrich, T., Kluppelberg, C., Adler, R.J.: Parameter estimation for ARMA models with infinite variance innovations. Ann. Stat. 23(1), 305-326 (1995)

11. Klüppelberg, C., Mikosch, T., et al.: The integrated periodogram for stable processes. Ann. Stat. 24(5), 1855-1879 (1996)

12. Gallagher, C.M.: A method for fitting stable autoregressive models using the autocovariation function. Stat. Probab. Lett. 53, 381-390 (2001)

13. Andrews, B., Calder, M., Davis, R.A.: Maximum likelihood estimation for $\alpha$-stable autoregressive processes. Ann. Stat. 37(4), 1946-1982 (2009)

14. Hannsgen, G.: Do the innovations in a monetary VAR have finite variances? Working Paper 546, The Levy Economics Institute of Bard College (2008)

15. Bielak, Ł., Grzesiek, A., Janczura, J., Wyłomańska, A.: Market risk factors analysis for an international mining company. Multidimensional, heavy-tailed-based modelling (2021). hyperimagehttp://arxiv.org/abs/2107.07142arXiv:2107.07142

16. Lévy, P.: Calcul des Probabilites. Gauthier-Villars, Paris (1925)

17. Khinchine, A.Y., Lévy, P.: Sur les lois stables. Comptes rendus de l'Académie des Sciences 202, 374-376 (1936)

18. Gnedenko, B., Kolomogorov, A.N.: Limit Distribution for Sums of Independent Random Variables. Addison-Wesley, New York (1954)

19. Feller, W.: An Introduction to Probability Theory and Its Applications, vol. II. John Wiley \& Sons Inc., London (1971). (Second edition)

20. Zolotarev, V.: One-dimensional Stable Distributions. Translations of mathematical monographs. American Mathematical Society (1986)

21. Weron, A.: Stable processes and measures; a survey. In: D. Szynal, A. Weron (eds.) Probability Theory on Vector Spaces III, pp. 306-364. Springer (1984)

22. Shao, M., Nikias, L., C. : Signal processing with fractional lower order moments: Stable processes and their application. Proceedings of the IEEE 81, 986-1010 (1993)

23. Janicki, A., Weron, A.: Simulation and Chaotic Behavior of $\alpha$ Stable Stochastic Processes. Marcel Dekker, New York (1994)

24. Samorodnitsky, G., Taqqu, M.: Stable Non-Gaussian Random Processes: Stochastic Models with Infinite Variance. Chapman \& Hall, New York (1994)

25. Mandelbrot, B.: The Pareto-Lévy Law and the distribution of income. International Economic Review 1(2), 79-106 (1960)

26. McCulloch, J.H.: 13 Financial applications of stable distributions. In: Statistical Methods in Finance, Handbook of Statistics, vol. 14, pp. 393-425. Elsevier (1996)

27. Bidarkota, P.V., McCulloch, J.H.: Optimal univariate inflation forecasting with symmetric stable shocks. J. Appl. Econ. 13(6), 659-670 (1998)

28. Mittnik, S., Rachev, S.T.: Stable Paretian Models in Finance. Wiley, London (2000) 
29. Belkacem, L., Véhel, J.L., Walter, C.: Capm, risk and portfolio selection in $\alpha$-stable markets. Fractals 8(1), 99-115 (2000)

30. Nolan, J.P.: Modeling financial data with stable distributions. In: Rachev, S.T. (ed.) Handbook of Heavy Tailed Distributions in Finance, Handbooks in Finance, vol. 1, pp. 105-130. NorthHolland (2003)

31. Rodriguez-Aguilar, R., Marmolejo-Saucedo, J.A., RetanaBlanco, B.: Prices of Mexican Wholesale Electricity Market: An Application of Alpha-Stable Regression. Sustainability 11(11), 1-14 (2019)

32. Molina-Muñoz, J., Mora-Valencia, A., Perote, J.: Market-crash forecasting based on the dynamics of the alpha-stable distribution. Phys. A: Statistical Mechanics and its Applications 557, 124876 (2020)

33. Kakinaka, S., Umeno, K.: Characterizing cryptocurrency market with Lévy's stable distributions. J. Phys. Soc. Jp 89(2), 024802 (2020)

34. Bendler, J.: Lévy (stable) probability densities and mechanical relaxation in solid polymers. J. Stat. Phys. 36, 625-637 (1984)

35. Weron, A., Weron, K.: Stable measures and processes in statistical physics. In: Probability in Banach Spaces V. Lecture Notes in Mathematics, vol. 1153, pp. 440-452. Springer (1985)

36. Kincses, D.: Shape Analysis of HBT Correlations at STAR. Physics of Particles and Nuclei 51, 267-269 (2020)

37. Nikias, C., Shao, M.: Signal Processing with AlphaStable Distributions and Applications. Signal Processing, Learning, Communications and Control. Wiley, Adaptive and Cognitive Dynamic Systems (1995)

38. Stuck, B.W.: An historical overview of stable probability distributions in signal processing. In: 2000 IEEE International Conference on Acoustics, Speech, and Signal Processing. Proceedings (Cat. No.00CH37100), vol. 6, pp. 3795-3797 (2000)

39. Jaoua, N., Duflos, E., Vanheeghe, P., Clavier, L., Septier, F.: Joint estimation of state and noise parameters in a linear dynamic system with impulsive measurement noise: application to OFDM systems. Digital Signal Process. 35, 21-36 (2014)

40. Simmross-Wattenberg, F., Asensio-Perez, J.I., Casaseca-de-la Higuera, P., Martin-Fernandez, M., Dimitriadis, I.A., AlberolaLopez, C.: Anomaly detection in network traffic based on statistical inference and $\alpha$-stable modeling. IEEE Trans. Depend. Secure Comput. 8(4), 494-509 (2011)

41. Obuchowicz, A.K., Smołka, M.: Application of $\alpha$-stable mutation in a hierarchic evolutionary inverse solver. J. Comput. Sci. 17(P1), 261-269 (2016)

42. Painter, S., Beresford, G., Paterson, L.: On the distribution of seismic reflection coefficients and seismic amplitudes. Geophysics 60(4), 1187-1194 (1995)

43. Chave, A.D.: Magnetotelluric data, stable distributions and impropriety: an existential combination. Geophys. J. Int. 198(1), 622-636 (2014)

44. Salas-Gonzalez, D., Kuruoglu, E.E., Ruiz, D.P.: Modelling and assessing differential gene expression using the alpha-stable distribution. Int. J.Biostat. 5(1), 1-16 (2009)

45. Lan, B.L., Chandran, P.: Distribution of animal population fluctuations. Phys. A Stat. Mech. Appl. 390(7), 1289-1294 (2011)

46. Miller, G.: Properties of certain symmetric stable distributions. J. Multivar. Anal. 8(3), 346-360 (1978)

47. Cambanis, S., Miller, G.: Linear problems in pth order and stable processes. J. Appl. Math. 41(1), 43-69 (1981)

48. Rosadi, D., Deistler, M.: Estimating the codifference function of linear time series models with infinite variance. Metrika 73(3), 395-429 (2011)

49. Rosadi, D.: Testing for independence in heavy-tailed time series using the codifference function. Comput. Stat. Data Anal. 53(12), 4516-4529 (2009)
50. Wyłomańska, A., Chechkin, A., Gajda, J., Sokolov, I.M.: Codifference as a practical tool to measure interdependence. Phys. A Stat. Mech. Appl. 421, 412-429 (2015)

51. Press, S.: Multivariate stable distributions. J. Multivar. Anal. 2(4), 444-462 (1972)

52. Paulauskas, V.: Some remarks on multivariate stable distributions. J. Multivar. Anal. 6(3), 356-368 (1976)

53. d'Estampes, L., Garel, B., Tjøstheim, D.: On a New Measure of Covariation for Stable Random Variables. Science Direct Working Paper No S1574-0358(04)70334-2 https://ssrn.com/abstract=3142692, 1-13 (2002)

54. Garel, B., d'Estampes, L., Tjøstheim, D.: Revealing some unexpected dependence properties of linear combinations of stable random variables using symmetric covariation. Commun. Stat. Theory Methods 33(4), 769-786 (2005)

55. Garel, B., Kodia, B.: Signed symmetric covariation coefficient for alpha-stable dependence modeling. Compt. Rend. Math. 347(5-6), 315-320 (2009)

56. Kodia, B., Garel, B.: Estimation and comparison of signed symmetric covariation coefficient and generalized association parameter for alpha-stable dependence modeling. Commun. Stat. Theory Methods 43(24), 5156-5174 (2014)

57. Ma, Xinyu, Nikias, C.L.: Joint estimation of time delay and frequency delay in impulsive noise using fractional lower order statistics. IEEE Trans. Signal Process. 44(11), 2669-2687 (1996)

58. Eliazar, I., Klafter, J.: Correlation cascades of Lévy-driven random processes. Phys. A Stat. Mech. Appl. 376, 1-26 (2007)

59. Magdziarz, M.: Correlation cascades, ergodic properties and long memory of infinitely divisible processes. Stochastic Processes Appl. 119(10), 3416-3434 (2009)

60. Garel, B.: Measures of dependence for infinite variance distributions. In: V. Melas, S. Mignani, P. Monari, L. Salmaso (eds.) Topics in statistical simulation, pp. 209-216. Springer (2014)

61. Alparslan, U., Nolan, J.: A measure of dependence for stable distributions. Extremes 19, 303-323 (2016)

62. Astrauskas, A., Lévy, J.B., Taqqu, M.: The asymptotic dependence structure of the linear fractional Lévy motion. Lithuanian Math. J. 31, 1-19 (1991)

63. Kokoszka, P.S., Taqqu, M.S.: Fractional ARIMA with stable innovations. Stochastic Process. Appl. 60(1), 19-47 (1995)

64. Lévy, J.B., Taqqu, M.S.: Dependence structure of a renewalreward process with infinite variance. Fractals 09(02), 185-192 (2001)

65. Maejima, M., Yamamoto, K.: Long-memory stable OrnsteinUhlenbeck processes. Electron. J. Probabil. 8(19), 1-18 (2003)

66. Lévy, J.B., Taqqu, M.S.: The asymptotic codifference and covariation of log-fractional stable noise. J. Econ. 181(1), 34-43 (2014)

67. Lévy, J.B., Taqqu, M.S.: The long-range dependence of linear log-fractional stable motion. Commun. Stochastic Anal.- 5, 187-210 (2011)

68. Nowicka-Zagrajek, J., Wyłomańska, A.: The dependence structure for PARMA models with alpha-stable innovations. Acta Phys. Pol. 37(1), 3071-3081 (2006)

69. Nowicka-Zagrajek, J., Wyłomańska, A.: Measures of dependence for stable AR(1) models with time-varying coefficients. Stochastic Models 24(1), 58-70 (2008)

70. Grzesiek, A., Teuerle, M., Wyłomańska, A.: Cross-codifference for bidimensional $\operatorname{VAR}(1)$ time series with infinite variance. Commun.Stat. Simul. $\quad$ Comput. https://doi.org/10.1080/03610918.2019.1670840

71. Grzesiek, A., Sikora, G., Teuerle, M., Wyłomańska, A.: Spatiotemporal dependence measures for bivariate AR(1) models with $\alpha$-stable noise. J. Time Ser. Anal. 41(3), 454-475 (2020) 
72. Grzesiek, A., Wyłomańska, A.: Asymptotic behavior of the crossdependence measures for bidimensional AR(1) model with $\alpha$ stable noise. Banach Center Publ. 122, 133-157 (2020)

73. Grzesiek, A., Wyłomańska, A.: Asymptotics of alternative interdependence measures for bivariate $\alpha$-stable autoregressive model of order 1. In: F. Chaari, J. Leskow, A. Wyłomańska, R. Zimroz (eds.) Nonstationary Systems: Theory and Applications. WNSTA 2021. Applied Condition Monitoring, vol. 18, pp. 41-68. Springer (2022)

74. Grzesiek, A., Giri, P., Sundar, S., Wyłomańska, A.: Measures of cross-dependence for bidimensional periodic AR(1) model with $\alpha$-stable distribution. J. Time Ser. Anal. 41(6), 785-807 (2020)

75. Nolan, J.P.: Multivariate stable distributions: Approximation, estimation, simulation and identification. In: Adler, R.J.,
Feldman, R.E., Taqqu, M.S. (eds.) A Practical Guide to Heavy Tails: Statistical Techniques and Applications, pp. 509-526. Birkhäuser Boston (1998)

76. Nolan, J.P.: An overview of multivariate stable distributions (2008). http://academic2.american.edu//stable/overview.pdf

77. Kruczek, P., Wyłomańska, A., Teuerle, M., Gajda, J.: The modified Yule-Walker method for $\alpha$-stable time series models. Phys. A 469, 588-603 (2017)

Publisher's Note Springer Nature remains neutral with regard to jurisdictional claims in published maps and institutional affiliations. 